\title{
Enriched finite element subspaces for dual-dual mixed formulations in fluid mechanics and elasticity ${ }^{\star}$
}

\author{
Rommel Bustinza ${ }^{a}$, Gabriel N. Gatica ${ }^{\text {a }}$, María González ${ }^{\text {b, }}$ \\ Salim Meddahi ${ }^{\mathrm{c}}$ and Ernst P. Stephan ${ }^{\mathrm{d}}$ \\ ${ }^{\mathrm{a}} \mathbf{G} \mathbf{I}^{2} \mathbf{M A}$, Departamento de Ingeniería Matemática, Universidad de Concepción, \\ Casilla 160-C, Concepción, Chile, e-mails: rbustinz/ggatica@ing-mat.udec.cl \\ ${ }^{\mathrm{b}}$ Departamento de Matemáticas, Universidade da Coruña, 15071 A Coruña, \\ España, e-mail: mgtaboad@udc.es \\ ${ }^{\mathrm{c}}$ Departamento de Matemáticas, Universidad de Oviedo, Calvo Sotelo s/n, Oviedo, \\ España, e-mail: salim@orion.ciencias.uniovi.es \\ ${ }^{\mathrm{d}}$ Institut für Angewandte Mathematik, Universität Hannover, Welfengarten 1, \\ D-30167 Hannover, Germany, e-mail: stephan@ifam.uni-hannover.de
}

\begin{abstract}
In this paper we unify the derivation of finite element subspaces guaranteeing unique solvability and stability of the Galerkin schemes for a new class of dual-mixed variational formulations. The approach, which has been applied to several linear and nonlinear boundary value problems, is based on the introduction of additional unknowns given by the flux and the gradient of velocity, and by the stress and strain tensors, for fluid mechanics and elasticity problems, respectively. In this way, the procedure yields two-fold saddle point operator equations as the resulting weak formulations (also named dual-dual ones), which are analyzed by means of a slight generalization of the well known Babuška-Brezzi theory. Then, in order to introduce well posed Galerkin schemes, we extend the corresponding proofs of the continuous formulations to the discrete schemes, and show that some usual finite elements need to be suitable enriched, depending on the nature of the problem. This leads to piecewise constant functions, Raviart-Thomas of lowest order, PEERS elements, and the deviators of them, as the appropriate subspaces.
\end{abstract}

Key words: two-fold saddle point, Raviart-Thomas, PEERS, enriched subspaces

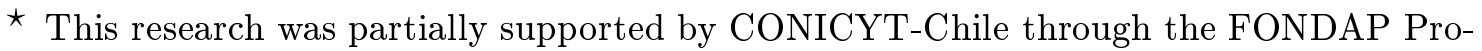
gram in Applied Mathematics, and by the Dirección de Investigación of the Universidad de Concepción through the Advanced Research Groups Program. 


\section{Introduction}

In a series of recent papers we have analyzed a new class of dual-mixed variational formulations for several boundary value problems arising in physics and engineering sciences (see [4], [16], [11], [12] and [6]). In particular, in [16] we consider the nonlinear incompressible material from [7] and apply the mixed approach first employed in [13] and [4], to study the solvability and Galerkin approximations of the associated variational formulation. Our approach there follows [4] and introduces the strain tensor and the rotation as additional unknowns, which yields a two-fold saddle point operator equation as the corresponding weak formulation. Hence, the abstract theory developed in [9], which is a slight generalization of the well known Babuška-Brezzi theory, is applied to prove that the continuous and discrete schemes are well posed. In particular, it is shown that the unique solvability and stability of the Galerkin scheme is guaranteed if the finite element subspaces are given by a suitable enrichment of the well known PEERS elements (cf. [1]).

On the other hand, in [11] we introduce and analyze a dual-mixed formulation for a class of quasi-Newtonian Stokes flows whose kinematic viscosities are nonlinear monotone functions of the gradient of the velocity. The mixed finite element method proposed there now relies on the introduction of the flux and the tensor gradient of the velocity as auxiliary unknowns, which also yields a two-fold saddle point operator equation as the resulting variational formulation. The rest of the analysis, although similar, is simpler than the one developed in [16]. In this case we show that, in order to guarantee that the Galerkin scheme is well posed, it suffices to use Raviart-Thomas spaces of order zero to approximate the flux and piecewise constant functions to approximate the other unknowns. Furthermore, since the monotonicity includes the linear case, we also obtain as a by-product a new mixed finite element method for the linear Stokes equation.

Next, in [6] we extend the analysis from [16] and [11] to the generalized Stokes problem. This is a Stokes-like linear system with a dominating zeroth order term, which plays a fundamental role in the numerical simulation of viscous incompressible flows. The approach here, being mainly a natural extension of the procedure applied in [11], introduces again the flux and the tensor gradient of the velocity as further unknowns. In addition, the resulting variational formulation also shows a two-fold saddle point structure, and hence the above mentioned generalized Babuška-Brezzi theory is applied to prove that the continuous and Galerkin schemes are well posed. In particular, the corresponding finite element subspaces coincide with those derived in [11] except for the one approximating the gradient of the velocity, which needs to be suitably enriched.

The purpose of the present paper is to provide a unified treatment for the derivation of the finite element subspaces yielding unique solvability and stability of the Galerkin schemes studied in [16], [11] and [6]. More precisely, we show in each case 
that these subspaces arise naturally from an inspection of the conditions required to extend to the discrete schemes the proofs of the unique solvability and stability of the continuous formulations. In the following three sections we provide the details for the boundary value problems from [11], [6] and [16], respectively, which corresponds to an increasing degree of enrichment of the resulting subspaces. Finally, in Section 5 we summarize the main results of our analysis and provide some further remarks.

In what follows, given any Hilbert space $H$, we denote by $H^{2}$ and $H^{2 \times 2}$ the spaces of vectors and tensors of order two, respectively, with entries in $H$, provided with the product norms induced by the norm of $H$. In addition, for any $\boldsymbol{\tau}:=\left(\tau_{i j}\right), \boldsymbol{\zeta}:=$ $\left(\zeta_{i j}\right) \in \mathbb{R}^{2 \times 2}$, we denote $\operatorname{tr}(\boldsymbol{\tau}):=\tau_{11}+\tau_{22}$ and $\boldsymbol{\tau}: \boldsymbol{\zeta}:=\sum_{i, j=1}^{2} \tau_{i j} \zeta_{i j}$. The deviator of tensor $\boldsymbol{\tau}$ is denoted by $\operatorname{dev}(\boldsymbol{\tau}):=\boldsymbol{\tau}-\frac{1}{2} \operatorname{tr}(\boldsymbol{\tau}) \mathbf{I}$, which satisfies $\operatorname{tr}(\operatorname{dev}(\boldsymbol{\tau}))=0$. We also use ${ }^{t}$ to denote the transpose of vectors and tensors.

\section{A nonlinear Stokes problem}

This section deals with the nonlinear boundary value problem studied in [11]. We first let $\Omega$ be a bounded and simply connected domain in $\mathbb{R}^{2}$ with Lipschitzcontinuous boundary $\Gamma$. Thus, we are interested in determining the velocity $\mathbf{u}:=$ $\left(u_{1}, u_{2}\right)^{t}$ and the pressure $p$ of a nonlinear Stokes fluid occupying the region $\Omega$ under the action of an external force. More precisely, given $\mathbf{f} \in\left[L^{2}(\Omega)\right]^{2}$ and $\mathbf{g} \in\left[H^{1 / 2}(\Gamma)\right]^{2}$, we look for $(\mathbf{u}, p)$ in appropriate spaces such that

$$
\begin{gathered}
-\operatorname{div}(\psi(|\nabla \mathbf{u}|) \nabla \mathbf{u}-p \mathbf{I})=\mathbf{f} \text { in } \Omega, \\
\operatorname{div}(\mathbf{u})=0 \text { in } \Omega, \text { and } \mathbf{u}=\mathbf{g} \text { on } \Gamma,
\end{gathered}
$$

where $\mathbf{d i v}$ and div are the usual vector and scalar divergence operators, $\nabla \mathbf{u}$ is the tensor gradient of $\mathbf{u},|\cdot|$ is the euclidean norm of $\mathbb{R}^{2}, \mathbf{I}$ is the identity matrix of $\mathbb{R}^{2 \times 2}$, and $\psi: \mathbb{R}^{+} \rightarrow \mathbb{R}^{+}$is the nonlinear kinematic viscosity function of the fluid. We remark that $\mathbf{g} \in\left[H^{1 / 2}(\Gamma)\right]^{2}$ must satisfy the compatibility condition $\int_{\Gamma} \mathbf{g} \cdot \mathbf{n} d s=0$, where $\mathbf{n}$ is the unit outward normal to $\Gamma$.

The kind of nonlinear Stokes problem given by (1) appears in the modeling of a large class of non-Newtonian fluids (see, e.g. [3], [17], [18], [20]). For instance, the Carreau law for viscoplastic flows (see, e.g. [18], [20]) reads $\psi(t):=\kappa_{0}+\kappa_{1}\left(1+t^{2}\right)^{(\beta-2) / 2}$ $\forall t \in \mathbb{R}^{+}$, with $\kappa_{0} \geq 0, \kappa_{1}>0$, and $\beta \geq 1$.

Throughout this Section we assume that $\psi$ is of class $C^{1}$ and that there exist $C_{1}$, $C_{2}>0$ such that for all $\mathbf{r}:=\left(r_{i j}\right), \mathbf{s}:=\left(s_{i j}\right) \in \mathbb{R}^{2 \times 2}$, there holds

$$
\left|\psi(|\mathbf{r}|) r_{i j}\right| \leq C_{1}\|\mathbf{r}\|_{\mathbb{R}^{2 \times 2}}, \quad\left|\frac{\partial}{\partial r_{k l}}\left\{\psi(|\mathbf{r}|) r_{i j}\right\}\right| \leq C_{1} \quad \forall i, j, k, l \in\{1,2\}
$$


and

$$
\sum_{i, j, k, l=1}^{2} \frac{\partial}{\partial r_{k l}}\left\{\psi(|\mathbf{r}|) r_{i j}\right\} s_{i j} s_{k l} \geq C_{2}\|\mathbf{s}\|_{\mathbb{R}^{2 \times 2}}^{2}
$$

The Carreau law satisfies (2) and (3) for all $\kappa_{0}>0$, and for all $\beta \in[1,2]$. In particular, $\beta=2$ yields the usual linear Stokes model.

We now introduce $\boldsymbol{\sigma}:=\psi(|\nabla \mathbf{u}|) \nabla \mathbf{u}-p \mathbf{I}$ and $\mathbf{t}:=\nabla \mathbf{u}$ in $\Omega$ as additional unknowns, and define the tensor $\boldsymbol{\psi}: \mathbb{R}^{2 \times 2} \rightarrow \mathbb{R}^{2 \times 2}$ by $\boldsymbol{\psi}(\mathbf{r}):=\left(\psi(|\mathbf{r}|) r_{i j}\right)$ for all $\mathbf{r} \in \mathbb{R}^{2 \times 2}$. In this way, the nonlinear constitutive law and the equilibrium equation become, respectively,

$$
\boldsymbol{\sigma}=\boldsymbol{\psi}(\mathbf{t})-p \mathbf{I} \quad \text { and } \quad \boldsymbol{d i v} \boldsymbol{\sigma}=-\mathbf{f} \quad \text { in } \Omega .
$$

In addition, since $\operatorname{div}(\mathbf{u})=\operatorname{tr}(\nabla \mathbf{u})$, the incompressibility condition can be rewritten as $\operatorname{tr}(\mathbf{t})=0$ in $\Omega$. Consequently, multiplying the relation $\mathbf{t}=\nabla \mathbf{u}$ by a tensor $\boldsymbol{\tau}$, integrating by parts, using that $\mathbf{u}=\mathbf{g}$ on $\Gamma$, testing appropriately the equations of (4) and the incompressibility of the fluid, and incorporating a suitable uniqueness condition (see [11] for details), we arrive at the following mixed variational formulation of (1): Find $(\mathbf{t},(\boldsymbol{\sigma}, p),(\mathbf{u}, \xi)) \in X_{1} \times M_{1} \times M$ such that

$$
\begin{array}{rcrl}
\int_{\Omega} \boldsymbol{\psi}(\mathbf{t}): \mathbf{s} & -\int_{\Omega} \boldsymbol{\sigma}: \mathbf{s}-\int_{\Omega} p \operatorname{tr}(\mathbf{s}) & =0 \\
-\int_{\Omega} \boldsymbol{\tau}: \mathbf{t}-\int_{\Omega} q \operatorname{tr}(\mathbf{t}) & -\int_{\Omega} \mathbf{u} \cdot \operatorname{div} \boldsymbol{\tau}+\xi \int_{\Omega} \operatorname{tr}(\boldsymbol{\tau}) & =-\langle\boldsymbol{\tau} \mathbf{n}, \mathbf{g}\rangle \\
-\int_{\Omega} \mathbf{v} \cdot \operatorname{div} \boldsymbol{\sigma}+\eta \int_{\Omega} \operatorname{tr}(\boldsymbol{\sigma}) & =\int_{\Omega} \mathbf{f} \cdot \mathbf{v}
\end{array}
$$

for all $(\mathbf{s},(\boldsymbol{\tau}, q),(\mathbf{v}, \eta)) \in X_{1} \times M_{1} \times M$, where the spaces are given by $X_{1}:=$ $\left[L^{2}(\Omega)\right]^{2 \times 2}, M_{1}:=H(\operatorname{div} ; \Omega) \times L^{2}(\Omega)$, and $M:=\left[L^{2}(\Omega)\right]^{2} \times \mathbb{R}$.

Hereafter, $\langle\cdot, \cdot\rangle$ denotes the duality pairing of $\left[H^{-1 / 2}(\Gamma)\right]^{2}$ and $\left[H^{1 / 2}(\Gamma)\right]^{2}$ with respect to the $\left[L^{2}(\Gamma)\right]^{2}$-inner product, and $H(\mathbf{d i v} ; \Omega)$ is the space of tensors $\boldsymbol{\tau} \in$ $\left[L^{2}(\Omega)\right]^{2 \times 2}$ satisfying $\operatorname{div}(\boldsymbol{\tau}) \in\left[L^{2}(\Omega)\right]^{2}$. It is well known that $H(\operatorname{div} ; \Omega)$, provided with the inner product $\langle\boldsymbol{\zeta}, \boldsymbol{\tau}\rangle_{H(\operatorname{div} ; \Omega)}:=\langle\boldsymbol{\zeta}, \boldsymbol{\tau}\rangle_{\left[L^{2}(\Omega)\right]^{2 \times 2}}+\langle\operatorname{div} \boldsymbol{\zeta}, \operatorname{div} \boldsymbol{\tau}\rangle_{\left[L^{2}(\Omega)\right]^{2}}$, is a Hilbert space, where $\langle\cdot, \cdot\rangle_{\left[L^{2}(\Omega)\right]^{2 \times 2}}$ and $\langle\cdot, \cdot\rangle_{\left[L^{2}(\Omega)\right]^{2}}$ stand for the usual inner products of $\left[L^{2}(\Omega)\right]^{2 \times 2}$ and $\left[L^{2}(\Omega)\right]^{2}$, respectively.

We also observe that one knows in advance that $\xi$, the Lagrange multiplier introduced by the uniqueness condition, vanishes. In fact, it suffices to take $\boldsymbol{\tau}=\mathbf{I}$ and $q=-1$ in the second equation of (5), and use the compatibility condition for the Dirichlet data g. However, this artificial unknown is needed to insure the symmetry of the whole formulation.

We now apply the abstract theory from [9] and [14] (see also [8]) to establish the main result concerning the solvability of (5).

THEOREM 2.1 There exists a unique $(\mathbf{t},(\boldsymbol{\sigma}, p),(\mathbf{u}, \xi)) \in X_{1} \times M_{1} \times M$ solution of 
problem (5). Moreover, there exists $C>0$, independent of the solution, such that

$$
\|(\mathbf{t},(\boldsymbol{\sigma}, p),(\mathbf{u}, \xi))\|_{X_{1} \times M_{1} \times M} \leq C\left\{\|\mathbf{f}\|_{\left[L^{2}(\Omega)\right]^{2}}+\|\mathbf{g}\|_{\left[H^{1 / 2}(\Gamma)\right]^{2}}\right\} .
$$

Proof. We begin by noticing that (5) has the two-fold saddle point structure studied in [9] and [14]. Indeed, let us define the operators $\mathbf{A}_{1}: X_{1} \rightarrow X_{1}^{\prime}, \mathbf{B}_{1}$ : $X_{1} \rightarrow M_{1}^{\prime}$, and $\mathbf{B}: M_{1} \rightarrow M^{\prime}$, and the functionals $(\mathbf{G}, \mathbf{F}) \in M_{1}^{\prime} \times M^{\prime}$, as follows

$$
\begin{gathered}
{\left[\mathbf{A}_{1}(\mathbf{r}), \mathbf{s}\right]:=\int_{\Omega} \boldsymbol{\psi}(\mathbf{r}): \mathbf{s} \quad, \quad\left[\mathbf{B}_{1}(\mathbf{r}),(\boldsymbol{\tau}, q)\right]:=-\int_{\Omega} \boldsymbol{\tau}: \mathbf{r}-\int_{\Omega} q \operatorname{tr}(\mathbf{r})} \\
{[\mathbf{B}(\boldsymbol{\tau}, q),(\mathbf{v}, \eta)]:=-\int_{\Omega} \mathbf{v} \cdot \operatorname{div}(\boldsymbol{\tau})+\eta \int_{\Omega} \operatorname{tr}(\boldsymbol{\tau})} \\
{[\mathbf{G},(\boldsymbol{\tau}, q)]:=-\langle\boldsymbol{\tau} \mathbf{n}, \mathbf{g}\rangle \quad \text { and } \quad[\mathbf{F},(\mathbf{v}, \eta)]:=\int_{\Omega} \mathbf{f} \cdot \mathbf{v}}
\end{gathered}
$$

for all $\mathbf{r}, \mathbf{s} \in X_{1},(\boldsymbol{\tau}, q) \in M_{1}$, and $(\mathbf{v}, \eta) \in M$, where $[\cdot, \cdot]$ stands for the duality pairing induced by the corresponding operators and functionals.

Then, it is easy to see that (5) can also be stated as: Find $(\mathbf{t},(\boldsymbol{\sigma}, p),(\mathbf{u}, \xi)) \in$ $X_{1} \times M_{1} \times M$ such that

$$
\begin{aligned}
{\left[\mathbf{A}_{1}(\mathbf{t}), \mathbf{s}\right]+\left[\mathbf{B}_{1}(\mathbf{s}),(\boldsymbol{\sigma}, p)\right] } & =0, \\
{\left[\mathbf{B}_{1}(\mathbf{t}),(\boldsymbol{\tau}, q)\right] } & +[\mathbf{B}(\boldsymbol{\tau}, q),(\mathbf{u}, \xi)]=[\mathbf{G},(\boldsymbol{\tau}, q)], \\
{[\mathbf{B}(\boldsymbol{\sigma}, p),(\mathbf{v}, \eta)] } &
\end{aligned}
$$

for all $(\mathbf{s},(\boldsymbol{\tau}, q),(\mathbf{v}, \eta)) \in X_{1} \times M_{1} \times M$.

The rest of the proof reduces to show that the formulation (9) satisfies the hypotheses of Theorem 2.4 in [9]. We first observe that for each $\tilde{\mathbf{r}} \in X_{1}$ the Gâteaux derivative $\mathcal{D} \mathbf{A}_{1}(\tilde{\mathbf{r}})$ is given by

$$
\mathcal{D A}_{1}(\tilde{\mathbf{r}})(\mathbf{r}, \mathbf{s})=\int_{\Omega}\left\{\sum_{i, j, k, l=1}^{2} \frac{\partial}{\partial \tilde{r}_{k l}} \psi_{i j}(\tilde{\mathbf{r}}) r_{k l} s_{i j}\right\} \quad \forall \mathbf{r}, \mathbf{s} \in X_{1}
$$

which, according to (2) and (3), becomes a uniformly bounded and uniformly $X_{1^{-}}$ elliptic bilinear form on $X_{1} \times X_{1}$. It is easy to see that these properties yield the strong monotonicity and Lipschitz continuity of the nonlinear operator $\mathbf{A}_{1}$.

We now check that the linear operator $\mathbf{B}$ verifies the inf-sup condition on $M_{1} \times M$. Given $(\mathbf{v}, \eta) \in M$, we have

$$
\sup _{\substack{\boldsymbol{\tau}, q) \in M_{1} \\(\boldsymbol{\tau}, q) \neq 0}} \frac{[\mathbf{B}(\boldsymbol{\tau}, q),(\mathbf{v}, \eta)]}{\|(\boldsymbol{\tau}, q)\|_{M_{1}}} \geq \frac{[\mathbf{B}(\eta \mathbf{I}, 0),(\mathbf{v}, \eta)]}{\|\eta \mathbf{I}\|_{H(\operatorname{div} ; \Omega)}}=(2|\Omega|)^{1 / 2}|\eta| .
$$


Next, using that $H(\boldsymbol{d i v} ; \Omega)=H_{0}(\boldsymbol{d i v} ; \Omega) \oplus \mathbb{R} \mathbf{I}$, where $H_{0}(\boldsymbol{d i v} ; \Omega):=\{\boldsymbol{\tau} \in$ $\left.H(\operatorname{div} ; \Omega): \quad \int_{\Omega} \operatorname{tr}(\boldsymbol{\tau})=0\right\}$, we deduce (see [11] for details) that

$$
\sup _{\substack{(\boldsymbol{\tau}, q) \in M_{1} \\(\boldsymbol{\tau}, q) \neq 0}} \frac{[\mathbf{B}(\boldsymbol{\tau}, q),(\mathbf{v}, \eta)]}{\|(\boldsymbol{\tau}, q)\|_{M_{1}}} \geq \sup _{\substack{\boldsymbol{\tau} \in H(\operatorname{div} ; \Omega) \\ \boldsymbol{\tau} \neq 0}} \frac{-\int_{\Omega} \mathbf{v} \cdot \operatorname{div}(\boldsymbol{\tau})}{\|\boldsymbol{\tau}\|_{H(\operatorname{div} ; \Omega)}}
$$

which, similarly as shown in Theorem 4.3 of [13], yields the existence of $\hat{\beta}>0$ such that

$$
\sup _{\substack{(\boldsymbol{\tau}, q) \in M_{1} \\(\boldsymbol{\tau}, q) \neq 0}} \frac{[\mathbf{B}(\boldsymbol{\tau}, q),(\mathbf{v}, \eta)]}{\|(\boldsymbol{\tau}, q)\|_{M_{1}}} \geq \hat{\beta}\|\mathbf{v}\|_{\left[L^{2}(\Omega)\right]^{2}}
$$

Therefore, (10) and (11) provide the continuous inf-sup condition for B.

We now introduce the null space of the operator $\mathbf{B}$, that is $\tilde{M}_{1}:=\left\{(\boldsymbol{\tau}, q) \in M_{1}\right.$ : $\operatorname{div}(\boldsymbol{\tau})=0$ in $\Omega$ and $\left.\int_{\Omega} \operatorname{tr}(\boldsymbol{\tau})=0\right\}$. It follows that there exists $\beta_{1}>0$ such that for all $(\boldsymbol{\tau}, q) \in \tilde{M}_{1}$ there holds

$$
\sup _{\substack{\mathbf{s} \in X_{1} \\ \mathbf{s} \neq 0}} \frac{\left[\mathbf{B}_{1}(\mathbf{s}),(\boldsymbol{\tau}, q)\right]}{\|\mathbf{s}\|_{X_{1}}} \geq \beta_{1}\|(\boldsymbol{\tau}, q)\|_{M_{1}}
$$

In fact, we prove (12), the continuous inf-sup condition for $\mathbf{B}_{1}$, by bounding below the expression $\sup _{\substack{\mathbf{s} \in X_{1} \\ \mathbf{s} \neq 0}} \frac{\left[\mathbf{B}_{1}(\mathbf{s}),(\boldsymbol{\tau}, q)\right]}{\|\mathbf{s}\|_{X_{1}}}$ with suitable choices of $\mathbf{s} \in X_{1}$. If $\|q\|_{L^{2}(\Omega)} \leq$ $\|\boldsymbol{\tau}\|_{H(\operatorname{div} ; \Omega)}$ we take $\mathbf{s}:=-\operatorname{dev}(\boldsymbol{\tau})$ and then apply that the norms $\|\boldsymbol{\tau}\|_{\left[L^{2}(\Omega)\right]^{2 \times 2}}$ and $\|\operatorname{dev}(\boldsymbol{\tau})\|_{\left[L^{2}(\Omega)\right]^{2 \times 2}}$ are equivalent for tensors $\boldsymbol{\tau} \in H(\operatorname{div} ; \Omega)$ satisfying $\int_{\Omega} \operatorname{tr}(\boldsymbol{\tau})=0$ (see Lemma 3.1 in [2]). Similarly, if $\|\boldsymbol{\tau}\|_{H(\operatorname{div} ; \Omega)} \leq\|q\|_{L^{2}(\Omega)}$ we just consider $\mathbf{s}:=$ $-q \mathbf{I}+\boldsymbol{\tau}$. We omit further details and refer the reader to Lemma 3.3 in [16] for a similar procedure.

Finally, noting that $\mathbf{A}_{1}(0)$ is the null functional, a straightforward application of Theorem 2.4 in [9] (see also Theorem 4.1 in [15]) completes the proof.

We now derive finite element subspaces yielding the Galerkin scheme associated with (9) to be well posed. To this end, we assume for simplicity that $\Gamma$ is a polygonal curve, and let $\left\{\mathcal{T}_{h}\right\}_{h>0}$ be a regular family of triangulations of $\bar{\Omega}$ by triangles $T$ of diameter $h_{T}$ such that $h:=\max \left\{h_{T}: T \in \mathcal{T}_{h}\right\}$ and $\bar{\Omega}=\cup\left\{T: T \in \mathcal{T}_{h}\right\}$. Then, we let $X_{1, h}, M_{1, h}^{\boldsymbol{\sigma}}, M_{1, h}^{p}$, and $M_{h}^{\mathbf{u}}$ be finite element subspaces for the unknowns $\mathbf{t}, \boldsymbol{\sigma}, p$, and $\mathbf{u}$, respectively, and define $M_{1, h}:=M_{1, h}^{\boldsymbol{\sigma}} \times M_{1, h}^{p}$ and $M_{h}:=M_{h}^{\mathbf{u}} \times \mathbb{R}$. According to Theorem 3.2 in [9], we now require the strong monotonicity and Lipschitz-continuity of $\mathbf{A}_{1}$ on $X_{1, h}$ and the discrete inf-sup conditions for $\mathbf{B}$ and $\mathbf{B}_{1}$. We show below that these properties follow by adapting the arguments of the continuous case.

Since the strong monotonicity and Lipschitz-continuity of $\mathbf{A}_{1}$ is clearly valid on any subspace of $X_{1}$, we realize that there is nothing else to prove for this nonlinear 
operator. Next, in order to extend the proof of the continuous inf-sup condition for B to the discrete case, we first require that $(\eta \mathbf{I}, 0)$ belongs to $M_{1, h}$ for any $\eta \in \mathbb{R}$, that is

$$
\eta \mathbf{I} \in M_{1, h}^{\boldsymbol{\sigma}} \quad \forall \eta \in \mathbb{R} .
$$

Secondly, since $M_{1, h}^{\boldsymbol{\sigma}}=\hat{M}_{1, h}^{\boldsymbol{\sigma}}+\mathbb{R} \mathbf{I}$, with $\hat{M}_{1, h}^{\boldsymbol{\sigma}}=M_{1, h}^{\boldsymbol{\sigma}} \cap H_{0}(\mathbf{d i v} ; \Omega)$, we also deduce that for all $(\mathbf{v}, \eta) \in M_{h}$ there holds

$$
\sup _{\substack{(\boldsymbol{\tau}, q) \in M_{1, h} \\(\boldsymbol{\tau}, q) \neq 0}} \frac{[\mathbf{B}(\boldsymbol{\tau}, q),(\mathbf{v}, \eta)]}{\|(\boldsymbol{\tau}, q)\|_{M_{1}}} \geq \sup _{\substack{\boldsymbol{\tau} \in M_{1, h} \\ \boldsymbol{\tau} \neq 0}} \frac{-\int_{\Omega} \mathbf{v} \cdot \operatorname{div} \boldsymbol{\tau}}{\|\boldsymbol{\tau}\|_{H(\operatorname{div} ; \Omega)}} .
$$

Then, in order to derive the existence of $\tilde{\beta}>0$, independent of $h$, such that

$$
\sup _{\substack{\boldsymbol{\tau}_{\in \rightarrow, h} \boldsymbol{\sigma} \\ \boldsymbol{\tau} \neq 0}} \frac{-\int_{\Omega} \mathbf{v} \cdot \operatorname{div} \boldsymbol{\tau}}{\|\boldsymbol{\tau}\|_{H(\operatorname{div} ; \Omega)}} \geq \tilde{\beta}\|\mathbf{v}\|_{\left[L^{2}(\Omega)\right]^{2}} \quad \forall \mathbf{v} \in M_{h}^{\mathbf{u}}
$$

we find, using the properties of the equilibrium interpolation operator (see, e.g. [5], [19]), as we did in Lemma 5.6 of [15], that it suffices to take

$$
M_{1, h}^{\boldsymbol{\sigma}}:=\left\{\boldsymbol{\tau} \in H(\operatorname{div} ; \Omega):\left.\quad \boldsymbol{\tau}\right|_{T} \in\left[\mathcal{R} \mathcal{T}_{0}(T)^{t}\right]^{2} \quad \forall T \in \mathcal{T}_{h}\right\}
$$

and

$$
M_{h}^{\mathbf{u}}:=\left\{\mathbf{v} \in\left[L^{2}(\Omega)\right]^{2}:\left.\quad \mathbf{v}\right|_{T} \in\left[\mathbf{P}_{0}(T)\right]^{2} \quad \forall T \in \mathcal{T}_{h}\right\}
$$

Hereafter, $\mathcal{R T}_{0}(T):=\left\langle\left(\begin{array}{l}1 \\ 0\end{array}\right),\left(\begin{array}{l}0 \\ 1\end{array}\right),\left(\begin{array}{l}x_{1} \\ x_{2}\end{array}\right)\right\rangle$ is the local Raviart-Thomas space of lowest order, and $\mathbf{P}_{0}(T)$ is the space of constant functions defined on $T$. It is easy to see that this choice of $M_{1, h}^{\boldsymbol{\sigma}}$ also guarantees the condition (13).

On the other hand, in order to prove the discrete inf-sup condition for $\mathbf{B}_{1}$, we need the discrete kernel of $\mathbf{B}$, which is given by $\tilde{M}_{1, h}:=\tilde{M}_{1, h}^{\boldsymbol{\sigma}} \times M_{1, h}^{p}$, where

$$
\tilde{M}_{1, h}^{\boldsymbol{\sigma}}:=\left\{\boldsymbol{\tau} \in M_{1, h}^{\boldsymbol{\sigma}}: \quad \operatorname{div} \boldsymbol{\tau}=0 \quad \text { in } \quad \Omega \quad \text { and } \quad \int_{\Omega} \operatorname{tr}(\boldsymbol{\tau})=0\right\}
$$

It follows, according to the choice of $M_{1, h}^{\boldsymbol{\sigma}}$, that the elements of $\tilde{M}_{1, h}^{\boldsymbol{\sigma}}$ are piecewise constant vectors on $\mathcal{T}_{h}$. In addition, it is clear that the norms $\|\boldsymbol{\tau}\|_{\left[L^{2}(\Omega)\right]^{2 \times 2}}$ and $\|\operatorname{dev}(\boldsymbol{\tau})\|_{\left[L^{2}(\Omega)\right]^{2 \times 2}}$ are also equivalent for each $\boldsymbol{\tau} \in \tilde{M}_{1, h}^{\boldsymbol{\sigma}}$. Hence, in order to extend the proof of the continuous inf-sup condition for $\mathbf{B}_{1}$ to the discrete case, we need to satisfy the following conditions:

$$
\operatorname{dev}(\boldsymbol{\tau}) \in X_{1, h} \quad \text { and } \quad(-q \mathbf{I}+\boldsymbol{\tau}) \in X_{1, h} \quad \forall(\boldsymbol{\tau}, q) \in \tilde{M}_{1, h} .
$$


The previous analysis does not impose any restriction on $M_{h}^{p}$, and therefore this subspace is choosen as the simplest possible one, that is as piecewise constant functions on the triangulation $\mathcal{T}_{h}$. According to the properties of $\tilde{M}_{1, h}^{\boldsymbol{\sigma}}$ and the choice of $M_{h}^{p}$, we observe that the requirements on $X_{1, h}$ given by (15) are satisfied if this subspace is taken as the piecewise constant vectors on $\mathcal{T}_{h}$, which is also the simplest possible one.

Consequently, the remaining finite element subspaces are given by

$$
X_{1, h}:=\left\{\mathbf{s} \in\left[L^{2}(\Omega)\right]^{2 \times 2}:\left.\mathbf{s}\right|_{T} \in\left[\mathbf{P}_{0}(T)\right]^{2 \times 2} \quad \forall T \in \mathcal{T}_{h}\right\},
$$

and

$$
M_{1, h}^{p}:=\left\{q \in L^{2}(\Omega):\left.\quad q\right|_{T} \in \mathbf{P}_{0}(T) \quad \forall T \in \mathcal{T}_{h}\right\}
$$

We are now in a position to establish the unique solvability and stability of the discrete problem, the Cea estimate, and the rate of convergence.

ThEOREM 2.2 The Galerkin scheme associated with the continuous problem (9) has a unique solution $\left(\mathbf{t}_{h},\left(\boldsymbol{\sigma}_{h}, p_{h}\right),\left(\mathbf{u}_{h}, \xi_{h}\right)\right) \in X_{1, h} \times M_{1, h} \times M_{h}$, and there exist positive constants $c, C$, independent of $h$, such that

$$
\left\|\left(\mathbf{t}_{h},\left(\boldsymbol{\sigma}_{h}, p_{h}\right),\left(\mathbf{u}_{h}, \xi_{h}\right)\right)\right\| \leq c\left\{\|\mathbf{f}\|_{\left[L^{2}(\Omega)\right]^{2}}+\|\mathbf{g}\|_{\left[H^{1 / 2}(\Gamma)\right]^{2}}\right\}
$$

and

$$
\begin{aligned}
& \left\|(\mathbf{t},(\boldsymbol{\sigma}, p),(\mathbf{u}, \xi))-\left(\mathbf{t}_{h},\left(\boldsymbol{\sigma}_{h}, p_{h}\right),\left(\mathbf{u}_{h}, \xi_{h}\right)\right)\right\| \\
& \leq C \inf _{\left(\mathbf{s}_{h},\left(\boldsymbol{\tau}_{h}, q_{h}\right), \mathbf{v}_{h}\right) \in X_{1, h} \times M_{1, h} \times M_{h}^{\mathbf{u}}}\left\|(\mathbf{t},(\boldsymbol{\sigma}, p), \mathbf{u})-\left(\mathbf{s}_{h},\left(\boldsymbol{\tau}_{h}, q_{h}\right), \mathbf{v}_{h}\right)\right\|,
\end{aligned}
$$

where $(\mathbf{t},(\boldsymbol{\sigma}, p),(\mathbf{u}, \xi))$ is the unique solution of $(9)$. In addition, under the assumption that $\mathbf{t} \in\left[H^{1}(\Omega)\right]^{2 \times 2}, \boldsymbol{\sigma} \in\left[H^{1}(\Omega)\right]^{2 \times 2}$, $\operatorname{div} \boldsymbol{\sigma} \in\left[H^{1}(\Omega)\right]^{2}, p \in H^{1}(\Omega)$, and $\mathbf{u} \in\left[H^{1}(\Omega)\right]^{2}$, there exists a positive constant $\bar{C}$, independent of $h$, such that

$$
\begin{aligned}
& \left\|(\mathbf{t},(\boldsymbol{\sigma}, p),(\mathbf{u}, \xi))-\left(\mathbf{t}_{h},\left(\boldsymbol{\sigma}_{h}, p_{h}\right),\left(\mathbf{u}_{h}, \xi_{h}\right)\right)\right\| \\
& \leq \bar{C} h\left\{\|\mathbf{t}\|_{\left[H^{1}(\Omega)\right]^{2 \times 2}}+\|\boldsymbol{\sigma}\|_{\left[H^{1}(\Omega)\right]^{2 \times 2}}+\|\operatorname{div} \boldsymbol{\sigma}\|_{\left[H^{1}(\Omega)\right]^{2}}+\|p\|_{H^{1}(\Omega)}+\|\mathbf{u}\|_{\left[H^{1}(\Omega)\right]^{2}}\right\} .
\end{aligned}
$$

Proof. We refer to Theorems 3.1 and 3.2 in [11] for details.

\section{The generalized Stokes problem}

In this section we deal with the boundary value problem analyzed in [6]. Let $\Omega$ be and simply connected domain in $\mathbb{R}^{2}$ with Lipschitz continuous boundary $\Gamma$. Then, 
given $\mathbf{f} \in\left[L^{2}(\Omega)\right]^{2}$ and $\mathbf{g} \in\left[H^{1 / 2}(\Gamma)\right]^{2}$, we look for the velocity $\mathbf{u}:=\left(u_{1}, u_{2}\right)^{t}$ and the pressure $p$ of a fluid occupying the region $\Omega$, such that

$$
\begin{gathered}
\alpha \mathbf{u}-\nu \Delta \mathbf{u}+\nabla p=\mathbf{f} \text { in } \Omega \\
\operatorname{div}(\mathbf{u})=0 \quad \text { in } \Omega, \quad \mathbf{u}=\mathbf{g} \text { on } \Gamma,
\end{gathered}
$$

where $\nu$ is a positive constant called kinematic viscosity of the fluid and $\alpha$ is a positive parameter proportional to the inverse of the time-step. Throughout the rest of the paper we assume that $\alpha \geq \nu$. Again, the incompressibility of the fluid requires the Dirichlet data $\mathbf{g}$ to satisfy the compatibility condition $\int_{\Gamma} \mathbf{g} \cdot \mathbf{n} d s=0$, where $\mathbf{n}$ is the unit outward normal to $\Gamma$.

We proceed as in Section 2 and introduce two additional unknowns in $\Omega$, namely, the tensor gradient of the velocity $\mathbf{t}:=\nabla \mathbf{u}$ and the flux $\boldsymbol{\sigma}:=\nu \nabla \mathbf{u}-p \mathbf{I}$, where $\mathbf{I}$ is the identity in $\mathbf{R}^{2 \times 2}$. It follows that the equilibrium equation becomes $\alpha \mathbf{u}-\operatorname{div}(\boldsymbol{\sigma})=$ $\mathbf{f}$ in $\Omega$, where $\boldsymbol{\sigma}:=\nu \mathbf{t}-p \mathbf{I}$. In addition, since $\operatorname{div}(\mathbf{u})=\operatorname{tr}(\mathbf{t})$ in $\Omega$, we can rewrite the incompressibility condition $\operatorname{as} \operatorname{tr}(\mathbf{t})=0$ in $\Omega$.

Now, let us define the spaces $X_{1}:=\left[L^{2}(\Omega)\right]^{2 \times 2} \times\left[L^{2}(\Omega)\right]^{2}, M_{1}:=H(\operatorname{div} ; \Omega)$, and $M:=L^{2}(\Omega) \times \mathbb{R}$. Then, following the usual procedure (see [6] for details), we obtain the following mixed variational formulation of $(16)$ : Find $((\mathbf{t}, \mathbf{u}), \boldsymbol{\sigma},(p, \xi)) \in$ $X_{1} \times M_{1} \times M$ such that

$$
\begin{array}{rlrl}
\nu \int_{\Omega} \mathbf{t}: \mathbf{s}+\alpha \int_{\Omega} \mathbf{u} \cdot \mathbf{v}-\int_{\Omega} \boldsymbol{\sigma}: \mathbf{s}-\int_{\Omega} \operatorname{div}(\boldsymbol{\sigma}) \cdot \mathbf{v}-\int_{\Omega} p \operatorname{tr}(\mathbf{s}) & =\int_{\Omega} \mathbf{f} \cdot \mathbf{v}, \\
-\int_{\Omega} \boldsymbol{\tau}: \mathbf{t}-\int_{\Omega} \operatorname{div}(\boldsymbol{\tau}) \cdot \mathbf{u} & +\xi \int_{\Omega} \operatorname{tr}(\boldsymbol{\tau}) & =-\langle\boldsymbol{\tau} \mathbf{n}, \mathbf{g}\rangle, \\
-\int_{\Omega} q \operatorname{tr}(\mathbf{t})+\eta \int_{\Omega} \operatorname{tr}(\boldsymbol{\sigma}) & =0,
\end{array}
$$

for all $((\mathbf{s}, \mathbf{v}), \boldsymbol{\tau},(q, \eta)) \in X_{1} \times M_{1} \times M$.

We now apply again the abstract theory from [9] and [14] (see also the related results given in [8]) to establish the solvability and continuous dependence of (17).

Theorem 3.1 Problem (17) has a unique solution $((\mathbf{t}, \mathbf{u}), \boldsymbol{\sigma},(p, \xi)) \in X_{1} \times M_{1} \times M$. Moreover, there exists a positive constant $C(\alpha, \nu)=O\left(\frac{\alpha^{3}}{\nu}\right)$, independent of the solution, such that

$$
\|((\mathbf{t}, \mathbf{u}), \boldsymbol{\sigma},(p, \xi))\|_{X_{1} \times M_{1} \times M} \leq C(\alpha, \nu)\left\{\|\mathbf{f}\|_{\left[L^{2}(\Omega)\right]^{2}}+\|\mathbf{g}\|_{\left[H^{1 / 2}(\Gamma)\right]^{2}}\right\} .
$$

ProOF. Similarly as in the proof of Theorem 2.1, we first observe that the mixed variational formulation (17) can be stated as the following two-fold saddle point 
operator equation: Find $((\mathbf{t}, \mathbf{u}), \boldsymbol{\sigma},(p, \xi)) \in X_{1} \times M_{1} \times M$ such that

$$
\begin{aligned}
& {\left[\mathbf{A}_{1}(\mathbf{t}, \mathbf{u}),(\mathbf{s}, \mathbf{v})\right]+\left[\mathbf{B}_{1}(\mathbf{s}, \mathbf{v}), \boldsymbol{\sigma}\right]+\left[\mathbf{B}^{p}(\mathbf{s}, \mathbf{v}), p\right]=[\mathbf{H},(\mathbf{s}, \mathbf{v})]} \\
& {\left[\mathbf{B}_{1}(\mathbf{t}, \mathbf{u}), \boldsymbol{\tau}\right]+\left[\mathbf{B}^{\xi}(\boldsymbol{\tau}), \xi\right]=[\mathbf{G}, \boldsymbol{\tau}]} \\
& {\left[\mathbf{B}^{p}(\mathbf{t}, \mathbf{u}), q\right]+\left[\mathbf{B}^{\xi}(\boldsymbol{\sigma}), \eta\right]=0,}
\end{aligned}
$$

for all $((\mathbf{s}, \mathbf{v}), \boldsymbol{\tau},(q, \eta)) \in X_{1} \times M_{1} \times M$, where the operators $\mathbf{A}_{1}: X_{1} \rightarrow X_{1}^{\prime}$, $\mathbf{B}_{1}: X_{1} \rightarrow M_{1}^{\prime}, \mathbf{B}^{p}: X_{1} \rightarrow L^{2}(\Omega)$, and $\mathbf{B}^{\xi}: M_{1} \rightarrow \mathbb{R}$, and the functionals $\mathbf{H} \in X_{1}^{\prime}$ and $\mathbf{G} \in M_{1}^{\prime}$, are defined as follows:

$$
\begin{gathered}
{\left[\mathbf{A}_{1}(\mathbf{r}, \mathbf{w}),(\mathbf{s}, \mathbf{v})\right]:=\nu \int_{\Omega} \mathbf{r}: \mathbf{s}+\alpha \int_{\Omega} \mathbf{w} \cdot \mathbf{v},} \\
{\left[\mathbf{B}_{1}(\mathbf{r}, \mathbf{w}), \boldsymbol{\tau}\right]:=-\int_{\Omega} \boldsymbol{\tau}: \mathbf{r}-\int_{\Omega} \operatorname{div}(\boldsymbol{\tau}) \cdot \mathbf{w}} \\
{\left[\mathbf{B}^{p}(\mathbf{r}, \mathbf{w}), q\right]:=-\int_{\Omega} q \operatorname{tr}(\mathbf{r}) \quad, \quad\left[\mathbf{B}^{\xi}(\boldsymbol{\zeta}), \eta\right]:=\eta \int_{\Omega} \operatorname{tr}(\boldsymbol{\zeta}),} \\
{[\mathbf{H},(\mathbf{s}, \mathbf{v})]:=\int_{\Omega} \mathbf{f} \cdot \mathbf{v}, \quad \text { and } \quad[\mathbf{G}, \boldsymbol{\tau}]:=-\langle\boldsymbol{\tau} \mathbf{n}, \mathbf{g}\rangle,}
\end{gathered}
$$

for all $(\mathbf{r}, \mathbf{w}),(\mathbf{s}, \mathbf{v}) \in X_{1}, \boldsymbol{\zeta}, \boldsymbol{\tau} \in M_{1}$ and $(q, \eta) \in M$. For further use, we also define the space $X:=X_{1} \times M_{1}$ and introduce the operators $\mathbf{A}: X \rightarrow X^{\prime}$ and $\mathbf{B}: X \rightarrow M^{\prime}$, which are given by

$$
[\mathbf{A}(\mathbf{r}, \mathbf{w}, \boldsymbol{\zeta}),(\mathbf{s}, \mathbf{v}, \boldsymbol{\tau})]:=\left[\mathbf{A}_{1}(\mathbf{r}, \mathbf{w}),(\mathbf{s}, \mathbf{v})\right]+\left[\mathbf{B}_{1}(\mathbf{r}, \mathbf{w}), \boldsymbol{\tau}\right]+\left[\mathbf{B}_{1}(\mathbf{s}, \mathbf{v}), \boldsymbol{\zeta}\right]
$$

and

$$
[\mathbf{B}(\mathbf{r}, \mathbf{w}, \boldsymbol{\zeta}),(q, \eta)]:=\left[\mathbf{B}^{p}(\mathbf{r}, \mathbf{w}), q\right]+\left[\mathbf{B}^{\xi}(\boldsymbol{\zeta}), \eta\right]
$$

Now, we observe that the operators $\mathbf{A}_{1}, \mathbf{B}_{1}$ and $\mathbf{B}$ are all linear and bounded. In particular, it is easy to see that $\left\|\mathbf{A}_{1}\right\|=O(\alpha)$ and that both $\left\|\mathbf{B}_{1}\right\|$ and $\|\mathbf{B}\|$ are of $O(1)$. In addition, since $\alpha \geq \nu$, we deduce from (20) that

$$
\left[\mathbf{A}_{1}(\mathbf{r}, \mathbf{w}),(\mathbf{r}, \mathbf{w})\right]=\nu\|\mathbf{r}\|_{\left[L^{2}(\Omega)\right]^{2 \times 2}}^{2}+\alpha\|\mathbf{w}\|_{\left[L^{2}(\Omega)\right]^{2}}^{2} \geq \nu\|(\mathbf{r}, \mathbf{w})\|_{X_{1}}^{2}
$$

for all $(\mathbf{r}, \mathbf{w}) \in X_{1}$, which shows that $\mathbf{A}_{1}$ is $X_{1}$-elliptic with an ellipticity constant given by $\nu$. Therefore, according to the linear version of Theorem 2.4 in [9] (see also Theorem 2 in [8]), it only remains to show that $\mathbf{B}$ and $\mathbf{B}_{1}$ satisfy the corresponding inf-sup conditions on $X \times M$ and on the kernel of $\mathbf{B}$, respectively.

Indeed, given $(q, \eta) \in M$ we take below $(\mathbf{s}, \mathbf{v}, \boldsymbol{\tau})=(0,0, \eta \mathbf{I})$ and $(\mathbf{s}, \mathbf{v}, \boldsymbol{\tau})=$ $(-q \mathbf{I}, 0,0)$, which are in $X$, to get

$$
\sup _{(\mathbf{s}, \mathbf{v}, \boldsymbol{\tau}) \in X} \frac{[\mathbf{B}(\mathbf{s}, \mathbf{v}, \boldsymbol{\tau}),(q, \eta)]}{\|(\mathbf{s}, \mathbf{v}, \boldsymbol{\tau})\|_{X}} \geq \frac{[\mathbf{B}(0,0, \eta \mathbf{I}),(q, \eta)]}{\|\eta \mathbf{I}\|_{\left[L^{2}(\Omega)\right]^{2 \times 2}}} \geq(2|\Omega|)^{1 / 2}|\eta|
$$


and

$$
\sup _{(\mathbf{s}, \mathbf{v}, \boldsymbol{\tau}) \in X} \frac{[\mathbf{B}(\mathbf{s}, \mathbf{v}, \boldsymbol{\tau}),(q, \eta)]}{\|(\mathbf{s}, \mathbf{v}, \boldsymbol{\tau})\|_{X}} \geq \frac{[\mathbf{B}(-q \mathbf{I}, 0,0),(q, \eta)]}{\sqrt{2}\|q\|_{L^{2}(\Omega)}} \geq \sqrt{2}\|q\|_{L^{2}(\Omega)}
$$

which prove the inf-sup condition for $\mathbf{B}$.

Next, we realize that the null space of the operator $\mathbf{B}$ is $\tilde{X}=\tilde{X}_{1} \times \tilde{M}_{1}$, where $\tilde{X}_{1}:=\left\{(\mathbf{s}, \mathbf{v}) \in X_{1}: \operatorname{tr}(\mathbf{s})=0 \quad\right.$ in $\left.\Omega\right\}$ and $\tilde{M}_{1}:=\left\{\boldsymbol{\tau} \in M_{1}: \int_{\Omega} \operatorname{tr}(\boldsymbol{\tau})=0\right\}$. Thus, given $\boldsymbol{\tau} \in \tilde{M}_{1}$ we take below $(\mathbf{s}, \mathbf{v})=(\mathbf{0},-\operatorname{div}(\boldsymbol{\tau}))$ and $(\mathbf{s}, \mathbf{v})=(-\operatorname{dev}(\boldsymbol{\tau}), \mathbf{0})$, which are in $\tilde{X}_{1}$, to obtain, respectively,

$$
\sup _{(\mathbf{s}, \mathbf{v}) \in \tilde{X}_{1}} \frac{\left[\mathbf{B}_{1}(\mathbf{s}, \mathbf{v}), \boldsymbol{\tau}\right]}{\|(\mathbf{s}, \mathbf{v})\|_{X_{1}}} \geq \frac{\left[\mathbf{B}_{1}(\mathbf{0},-\operatorname{div}(\boldsymbol{\tau})), \boldsymbol{\tau}\right]}{\|\operatorname{div}(\boldsymbol{\tau})\|_{\left[L^{2}(\Omega)\right]^{2}}}=\|\operatorname{div}(\boldsymbol{\tau})\|_{\left[L^{2}(\Omega)\right]^{2}}
$$

and

$$
\sup _{(\mathbf{s}, \mathbf{v}) \in \tilde{X}_{1}} \frac{\left[\mathbf{B}_{1}(\mathbf{s}, \mathbf{v}), \boldsymbol{\tau}\right]}{\|(\mathbf{s}, \mathbf{v})\|_{X_{1}}} \geq \frac{\left[\mathbf{B}_{1}(-\operatorname{dev}(\boldsymbol{\tau}), \mathbf{0}), \boldsymbol{\tau}\right]}{\|\operatorname{dev}(\boldsymbol{\tau})\|_{\left[L^{2}(\Omega)\right]^{2 \times 2}}}=\|\operatorname{dev}(\boldsymbol{\tau})\|_{\left[L^{2}(\Omega)\right]^{2 \times 2}} \geq C\|\boldsymbol{\tau}\|_{\left[L^{2}(\Omega)\right]^{2 \times 2}}
$$

where the last inequality makes use of the fact that the norms $\|\boldsymbol{\tau}\|_{\left[L^{2}(\Omega)\right]^{2 \times 2}}$ and $\|\operatorname{dev}(\boldsymbol{\tau})\|_{\left[L^{2}(\Omega)\right]^{2 \times 2}}$ are equivalent in $\tilde{M}_{1}$ (see Lemma 3.1 in [2]).

We end the proof by remarking that the analysis provided in Section 2 of [9] and a particular case of Proposition 2.3 in [21] (see Theorem 3.1 in [10]), yield the order of the continuous dependence constant $C(\alpha, \nu)$.

Our next goal is to derive finite element subspaces yielding a well posed Galerkin scheme. As in Section 2, we assume that $\Gamma$ is a polygonal curve, and let $\left\{\mathcal{T}_{h}\right\}_{h>0}$ be a regular family of triangulations of $\bar{\Omega}$ by triangles $T$ of diameter $h_{T}$ such that $h:=\max \left\{h_{T}: T \in \mathcal{T}_{h}\right\}$ and $\bar{\Omega}=\cup\left\{T: T \in \mathcal{T}_{h}\right\}$. Then, we let $X_{1, h}^{\mathbf{t}}, X_{1, h}^{\mathbf{u}}, M_{1, h}$, and $M_{h}^{p}$ be finite element subspaces for the unknowns $\mathbf{t}, \mathbf{u}, \boldsymbol{\sigma}$, and $p$, respectively, and define $X_{1, h}:=X_{1, h}^{\mathbf{t}} \times X_{1, h}^{\mathbf{u}}$ and $M_{h}:=M_{h}^{p} \times \mathbb{R}$. According to the linear version of Theorem 3.2 in [9], we now require the ellipticity of $\mathbf{A}_{1}$ on $X_{1, h}$ and the discrete inf-sup conditions for $\mathbf{B}$ and $\mathbf{B}_{1}$. Similarly as in Section 2 , we show below that these properties also follow by adapting the arguments of the continuous case.

The ellipticity of $\mathbf{A}_{1}$ is certainly valid on any subspace of $X_{1}$. Next, in order to extend the proof of the continuous inf-sup condition for $\mathbf{B}$ to the discrete case, we require that $(0,0, \eta \mathbf{I})$ and $(q \mathbf{I}, 0,0)$ belong to $X_{1, h}^{\mathbf{t}} \times X_{1, h}^{\mathbf{u}} \times M_{1, h}$ for any $(q, \eta) \in M_{h}^{p} \times \mathbb{R}$, that is

$$
\eta \mathbf{I} \in M_{1, h} \quad \forall \eta \in \mathbb{R} \quad \text { and } \quad q \mathbf{I} \in X_{1, h}^{\mathbf{t}} \quad \forall q \in M_{h}^{p}
$$

On the other hand, the proof of the discrete inf-sup condition for $\mathbf{B}_{1}$ needs the explicit knowledge of the discrete kernel of the bilinear form $\mathbf{B}$, which is given by 
$\tilde{X}_{1, h}^{\mathrm{t}} \times X_{1, h}^{\mathbf{u}} \times \tilde{M}_{1, h}$, where

$$
\tilde{X}_{1, h}^{\mathbf{t}}:=\left\{\mathbf{s} \in X_{1, h}^{\mathbf{t}}: \quad \int_{\Omega} q \operatorname{tr}(\mathbf{s})=0 \quad \forall q \in M_{h}^{p}\right\}
$$

and

$$
\tilde{M}_{1, h}:=\left\{\boldsymbol{\tau} \in M_{1, h}: \quad \int_{\Omega} \operatorname{tr}(\boldsymbol{\tau})=0\right\}
$$

It is important to observe here that $\tilde{M}_{1, h}$ is clearly a subspace of $\tilde{M}_{1}$ and hence the equivalence of $\|\boldsymbol{\tau}\|_{\left[L^{2}(\Omega)\right]^{2 \times 2}}$ and $\|\operatorname{dev}(\boldsymbol{\tau})\|_{\left[L^{2}(\Omega)\right]^{2 \times 2}}$ also holds for each $\boldsymbol{\tau} \in \tilde{M}_{1, h}$. Therefore, extending the proof of the continuous inf-sup condition for $\mathbf{B}_{1}$ to the discrete case, requires

$$
\operatorname{div}(\boldsymbol{\tau}) \in X_{1, h}^{\mathbf{u}} \quad \text { and } \quad \operatorname{dev}(\boldsymbol{\tau}) \in \tilde{X}_{1, h}^{\mathrm{t}} \quad \forall \boldsymbol{\tau} \in \tilde{M}_{1, h} .
$$

The equations (26) and (27) do not impose any explicit condition on the elements of $M_{h}^{p}$, and hence we choose this subspace of $L^{2}(\Omega)$ as the piecewise constant functions on the triangulation $\mathcal{T}_{h}$, which is the simplest possible one. Similarly, since the first restriction of (26) is satisfied if the piecewise constant tensors are included in $M_{1, h}$, we just choose this subspace of $H(\mathbf{d i v} ; \Omega)$ as the Raviart-Thomas space of order zero. According to this choice, and in order to satisfy the requirement on $X_{1, h}^{\mathbf{u}}$ given in (27), we realize that it suffices to take this subspace as the piecewise constant vectors on $\mathcal{T}_{h}$.

Now, taking into account the choices already made for $M_{h}^{p}$ and $M_{1, h}$, and observing that the trace of any deviator is zero, we find that the remaining conditions in (26) and (27) are accomplished if $X_{1, h}^{\mathbf{t}}$ is choosen so that its restriction on each triangle $T \in \mathcal{T}_{h}$ becomes the local space $\mathcal{D}_{0}(T):=\langle\mathbf{I}\rangle \oplus \operatorname{dev}\left(\left[\mathcal{R} \mathcal{T}_{0}(T)^{t}\right]^{2}\right)$, which, after straightforward computations, gives

$$
\begin{aligned}
& \mathcal{D}_{0}(T):=\left[\mathbf{P}_{0}(T)\right]^{2 \times 2} \oplus \operatorname{dev}\left(\left[\mathbf{P}_{0}(T) \mathbf{x}^{t}\right]^{2}\right) \\
& =\left[\mathbf{P}_{0}(T)\right]^{2 \times 2} \oplus\left\langle\left(\begin{array}{cc}
x_{1} & 2 x_{2} \\
0 & -x_{1}
\end{array}\right),\left(\begin{array}{cc}
-x_{2} & 0 \\
2 x_{1} & x_{2}
\end{array}\right)\right\rangle,
\end{aligned}
$$

where $\mathbf{x}^{t}:=\left(x_{1}, x_{2}\right)$ is the transpose of a generic vector $\mathbf{x} \in \mathbb{R}^{2}$.

As a consequence of the previous analysis, our finite element subspaces are

and

$$
\begin{aligned}
& X_{1, h}^{\mathrm{t}}:=\left\{\mathbf{s} \in\left[L^{2}(\Omega)\right]^{2 \times 2}:\left.\quad \mathbf{s}\right|_{T} \in \mathcal{D}_{0}(T) \quad \forall T \in \mathcal{T}_{h}\right\}, \\
& X_{1, h}^{\mathbf{u}}:=\left\{\mathbf{v} \in\left[L^{2}(\Omega)\right]^{2}:\left.\quad \mathbf{v}\right|_{T} \in\left[\mathbf{P}_{0}(T)\right]^{2} \quad \forall T \in \mathcal{T}_{h}\right\}, \\
& M_{1, h}:=\left\{\boldsymbol{\tau} \in H(\operatorname{div} ; \Omega):\left.\quad \boldsymbol{\tau}\right|_{T} \in\left[\mathcal{R} \mathcal{T}_{0}(T)^{t}\right]^{2} \quad \forall T \in \mathcal{T}_{h}\right\},
\end{aligned}
$$

$$
M_{h}^{p}:=\left\{q \in L^{2}(\Omega):\left.\quad q\right|_{T} \in \mathbf{P}_{0}(T) \quad \forall T \in \mathcal{T}_{h}\right\}
$$


Finally, the unique solvability and stability of the discrete problem, the corresponding Cea estimate, and the rate of convergence, are established in the following theorem.

THEOREM 3.2 The Galerkin scheme associated with the continuous problem (19) has a unique solution $\left(\left(\mathbf{t}_{h}, \mathbf{u}_{h}\right), \boldsymbol{\sigma}_{h},\left(p_{h}, \xi_{h}\right)\right) \in X_{1, h} \times M_{1, h} \times M_{h}$, and there exist positive constants $c, C=O\left(\frac{\alpha^{3}}{\nu}\right)$, independent of $h$, such that

$$
\left\|\left(\left(\mathbf{t}_{h}, \mathbf{u}_{h}\right), \boldsymbol{\sigma}_{h},\left(p_{h}, \xi_{h}\right)\right)\right\| \leq c\left(\|\mathbf{f}\|_{\left[L^{2}(\Omega)\right]^{2}}+\|\mathbf{g}\|_{\left[H^{1 / 2}(\Gamma)\right]^{2}}\right),
$$

and

$$
\begin{aligned}
& \left\|((\mathbf{t}, \mathbf{u}), \boldsymbol{\sigma},(p, \xi))-\left(\left(\mathbf{t}_{h}, \mathbf{u}_{h}\right), \boldsymbol{\sigma}_{h},\left(p_{h}, \xi_{h}\right)\right)\right\| \\
& \leq C \quad \inf _{\left(\left(\mathbf{s}_{h}, \mathbf{v}_{h}\right), \boldsymbol{\tau}_{h}, q_{h}\right) \in X_{1, h} \times M_{1, h} \times M_{h}^{p}}\left\|((\mathbf{t}, \mathbf{u}), \boldsymbol{\sigma}, p)-\left(\left(\mathbf{s}_{h}, \mathbf{v}_{h}\right), \boldsymbol{\tau}_{h}, q_{h}\right)\right\|,
\end{aligned}
$$

where $((\mathbf{t}, \mathbf{u}), \boldsymbol{\sigma},(p, \xi))$ is the unique solution of (19). In addition, under the assumption that $\mathbf{t} \in\left[H^{1}(\Omega)\right]^{2 \times 2}, \mathbf{u} \in\left[H^{1}(\Omega)\right]^{2}, \boldsymbol{\sigma} \in\left[H^{1}(\Omega)\right]^{2 \times 2}, \operatorname{div}(\boldsymbol{\sigma}) \in\left[H^{1}(\Omega)\right]^{2}$, and $p \in H^{1}(\Omega)$, there exists a positive constant $\bar{C}=O\left(\frac{\alpha^{3}}{\nu}\right)$, independent of $h$, such that

$$
\begin{aligned}
& \left\|((\mathbf{t}, \mathbf{u}), \boldsymbol{\sigma},(p, \xi))-\left(\left(\mathbf{t}_{h}, \mathbf{u}_{h}\right), \boldsymbol{\sigma}_{h},\left(p_{h}, \xi_{h}\right)\right)\right\| \\
& \leq \bar{C} h\left\{\|\mathbf{t}\|_{\left[H^{1}(\Omega)\right]^{2 \times 2}}+\|\boldsymbol{\sigma}\|_{\left[H^{1}(\Omega)\right]^{2 \times 2}}+\|\mathbf{u}\|_{\left[H^{1}(\Omega)\right]^{2}}+\|\operatorname{div}(\boldsymbol{\sigma})\|_{\left[H^{1}(\Omega)\right]^{2}}+\|p\|_{H^{1}(\Omega)}\right\} .
\end{aligned}
$$

Proof. We refer to Theorems 3.1 and 3.2 in [6] for details.

\section{A nonlinear elasticity problem}

This section deals with the problem from [16]. Again, we let $\Omega$ be a bounded and simply connected domain in $\mathbb{R}^{2}$ with Lipschitz-continuous boundary $\Gamma$. Our purpose now is to determine the displacement $\mathbf{u}:=\left(u_{1}, u_{2}\right)^{t}$ and the pressure-like unknown $p$ of a nonlinear incompressible material occupying the region $\Omega$ under the action of an external force. More precisely, given $\mathbf{f} \in\left[L^{2}(\Omega)\right]^{2}$ and $\mathbf{g} \in\left[H^{1 / 2}(\Gamma)\right]^{2}$, we look for $(\mathbf{u}, p)$ in appropriate spaces such that

$$
\begin{gathered}
-\operatorname{div}(\boldsymbol{\psi}(\mathbf{e}(\mathbf{u}))+p \mathbf{I})=\mathbf{f} \text { in } \Omega, \\
\operatorname{div}(\mathbf{u})=0 \text { in } \Omega, \text { and } \mathbf{u}=\mathbf{g} \text { on } \Gamma,
\end{gathered}
$$

where $\mathbf{e}(\mathbf{u})$ is the strain tensor of small deformations, and $\boldsymbol{\psi}: \mathbb{R}^{2 \times 2} \rightarrow \mathbb{R}^{2 \times 2}$ is a nonlinear mapping that induces a strongly monotone and Lipschitz-continuos ope- 
rator from $\left[L^{2}(\Omega)\right]^{2 \times 2}$ into its dual. A typical example of such a $\boldsymbol{\psi}$ is given by a hyperelastic material satisfying the Hencky-von Mises stress-strain relation (see, e.g. [13], [16]). We remark again that $\mathbf{g} \in\left[H^{1 / 2}(\Gamma)\right]^{2}$ must satisfy the condition $\int_{\Gamma} \mathbf{g} \cdot \mathbf{n} d s=0$, where $\mathbf{n}$ is the unit outward normal to $\Gamma$. Also, it is important to observe that the present boundary value problem (29) and the nonlinear Stokes model studied in Section 2 (cf. (1)) differ only on the fact that, instead of depending on $\nabla \mathbf{u}$, the nonlinear tensor $\boldsymbol{\psi}$ depends now on $\mathbf{e}(\mathbf{u})$. However, although the procedure is similar to the one employed in Section 2, this mere difference yields the introduction of another auxiliary unknown and the need of further enrichment of the finite element subspaces.

Indeed, we now introduce the strain tensor $\mathbf{t}:=\mathbf{e}(\mathbf{u})$, the stress tensor $\boldsymbol{\sigma}:=$ $\boldsymbol{\psi}(\mathbf{e}(\mathbf{u}))+p \mathbf{I}$, and the rotation $\boldsymbol{\gamma}:=\frac{1}{2}\left(\nabla \mathbf{u}-(\nabla \mathbf{u})^{t}\right)$ as additional unknowns. We notice that $\gamma$ lives in the subspace of $\left[L^{2}(\Omega)\right]^{2 \times 2}$ given by

$$
\mathcal{R}:=\left\{\boldsymbol{\delta} \in\left[L^{2}(\Omega)\right]^{2 \times 2}: \quad \boldsymbol{\delta}+\boldsymbol{\delta}^{t}=0\right\} .
$$

Then, following the usual procedure (see [16] for details), we arrive at the following mixed variational formulation of $(29)$ : Find $(\mathbf{t},(\boldsymbol{\sigma}, p),(\mathbf{u}, \boldsymbol{\gamma}, \xi)) \in X_{1} \times M_{1} \times M$ such that

$$
\begin{aligned}
\int_{\Omega} \boldsymbol{\psi}(\mathbf{t}): \mathbf{s} \quad-\int_{\Omega} \boldsymbol{\sigma}: \mathbf{s}+\int_{\Omega} p \operatorname{tr}(\mathbf{s}) & =0 \\
-\int_{\Omega} \boldsymbol{\tau}: \mathbf{t}+\int_{\Omega} q \operatorname{tr}(\mathbf{t})-\int_{\Omega} \mathbf{u} \cdot \operatorname{div} \boldsymbol{\tau}-\int_{\Omega} \boldsymbol{\gamma}: \boldsymbol{\tau}+\xi \int_{\Omega} \operatorname{tr}(\boldsymbol{\tau}) & =-\langle\boldsymbol{\tau} \mathbf{n}, \mathbf{g}\rangle \\
-\int_{\Omega} \mathbf{v} \cdot \operatorname{div} \boldsymbol{\sigma}-\int_{\Omega} \boldsymbol{\delta}: \boldsymbol{\sigma}+\eta \int_{\Omega} \operatorname{tr}(\boldsymbol{\sigma}) & =\int_{\Omega} \mathbf{f} \cdot \mathbf{v}
\end{aligned}
$$

for all $(\mathbf{s},(\boldsymbol{\tau}, q),(\mathbf{v}, \boldsymbol{\delta}, \eta)) \in X_{1} \times M_{1} \times M$, where the continuous spaces are given by $X_{1}:=\left[L^{2}(\Omega)\right]^{2 \times 2}, M_{1}:=H(\operatorname{div} ; \Omega) \times L^{2}(\Omega)$, and $M:=\left[L^{2}(\Omega)\right]^{2} \times \mathcal{R} \times \mathbb{R}$.

It is not difficult to see that (30) has the same two-fold saddle point structure obtained in Section 2 (cf. (9)). In particular, the corresponding linear operators $\mathbf{B}_{1}: X_{1} \rightarrow M_{1}^{\prime}$ and $\mathbf{B}: M_{1} \rightarrow M^{\prime}$ are defined by

$$
\left[\mathbf{B}_{1}(\mathbf{r}),(\boldsymbol{\tau}, q)\right]:=-\int_{\Omega} \boldsymbol{\tau}: \mathbf{r}+\int_{\Omega} q \operatorname{tr}(\mathbf{r})
$$

and

$$
[\mathbf{B}(\boldsymbol{\tau}, q),(\mathbf{v}, \boldsymbol{\delta}, \eta)]:=-\int_{\Omega} \mathbf{v} \cdot \operatorname{div}(\boldsymbol{\tau})-\int_{\Omega} \boldsymbol{\delta}: \boldsymbol{\tau}+\eta \int_{\Omega} \operatorname{tr}(\boldsymbol{\tau})
$$

for all $\mathbf{r} \in X_{1},(\boldsymbol{\tau}, q) \in M_{1}$, and $(\mathbf{v}, \boldsymbol{\delta}, \eta) \in M$. In addition, the unique solvability and stability of (30), being also a consequence of the abstract theory from [9] and [14], follow from similar arguments to those used in the proof of Theorem 2.1. We refer the interested reader to Section 3 in [16]. However, we do provide some more details below in the derivation of finite element subspaces leading to a well posed Galerkin scheme.

Indeed, as in Sections 2 and 3, we assume again that $\Gamma$ is a polygonal curve, and 
let $\left\{\mathcal{T}_{h}\right\}_{h>0}$ be a regular family of triangulations of $\bar{\Omega}$ by triangles $T$ of diameter $h_{T}$ such that $h:=\max \left\{h_{T}: T \in \mathcal{T}_{h}\right\}$ and $\bar{\Omega}=\cup\left\{T: T \in \mathcal{T}_{h}\right\}$. Then, we let $X_{1, h}, M_{1, h}^{\boldsymbol{\sigma}}$, $M_{1, h}^{p}, M_{h}^{\mathbf{u}}$, and $M_{h}^{\gamma}$ be finite element subspaces for the unknowns $\mathbf{t}, \boldsymbol{\sigma}, p, \mathbf{u}$, and $\boldsymbol{\gamma}$, respectively.

In order to extend the proof of the continuous inf-sup condition for $\mathbf{B}$ to the discrete case, we need that

$$
\eta \mathbf{I} \in M_{1, h}^{\boldsymbol{\sigma}} \quad \forall \eta \in \mathbb{R},
$$

and require the existence of $\tilde{\beta}>0$ such that for all $(\mathbf{v}, \boldsymbol{\delta}) \in M_{h}^{\mathbf{u}} \times M_{h}^{\gamma}$ there holds

$$
\sup _{\substack{\boldsymbol{\tau} \in M_{1, h} \boldsymbol{\sigma} \\ \boldsymbol{\tau} \neq 0}} \frac{-\int_{\Omega} \mathbf{v} \cdot \operatorname{div} \boldsymbol{\tau}-\int_{\Omega} \boldsymbol{\delta}: \boldsymbol{\tau}}{\|\boldsymbol{\tau}\|_{H(\operatorname{div} ; \Omega)}} \geq \tilde{\beta}\|(\mathbf{v}, \boldsymbol{\delta})\|_{\left[L^{2}(\Omega)\right]^{2} \times\left[L^{2}(\Omega)\right]^{2 \times 2}}
$$

Then, we recall from Lemma 4.4 in [1] that (34) is guaranteed by choosing

$$
\begin{gathered}
M_{1, h}^{\boldsymbol{\sigma}}:=\left\{\boldsymbol{\tau} \in H(\operatorname{div} ; \Omega):\left.\quad \boldsymbol{\tau}\right|_{T} \in\left[\mathcal{R}_{0}(T)^{t}\right]^{2} \oplus\left[\mathbf{P}_{0}(T) \operatorname{curl}^{t} b_{T}\right]^{2} \quad \forall T \in \mathcal{T}_{h}\right\}, \\
M_{h}^{\mathbf{u}}:=\left\{\mathbf{v} \in\left[L^{2}(\Omega)\right]^{2}:\left.\quad \mathbf{v}\right|_{T} \in\left[\mathbf{P}_{0}(T)\right]^{2} \quad \forall T \in \mathcal{T}_{h}\right\},
\end{gathered}
$$

and

$$
M_{h}^{\gamma}:=\left\{\boldsymbol{\delta} \in \mathcal{R} \cap\left[H^{1}(\Omega)\right]^{2 \times 2}:\left.\quad \boldsymbol{\delta}\right|_{T} \in\left[\mathbf{P}_{1}(T)\right]^{2 \times 2} \quad \forall T \in \mathcal{T}_{h}\right\},
$$

where $b_{T}$ is the usual cubic bubble function on the triangle $T \in \mathcal{T}_{h}$, $\operatorname{curl}^{t} b_{T}:=$ $\left(\frac{\partial b_{T}}{\partial x_{2}},-\frac{\partial b_{T}}{\partial x_{1}}\right)$, and $\mathbf{P}_{1}(T)$ denotes the space of polynomials of degree $\leq 1$ on $T$. The triple $M_{1, h}^{\boldsymbol{\sigma}} \times M_{h}^{\mathbf{u}} \times M_{h}^{\gamma}$ corresponds precisely to the PEERS finite element subspace approximating $(\boldsymbol{\sigma}, \mathbf{u}, \boldsymbol{\gamma})$. It is easy to see that (33) is clearly satisfied with this choice of $M_{1, h}^{\boldsymbol{\sigma}}$.

On the other hand, the discrete kernel of B is given by $\tilde{M}_{1, h}:=\tilde{M}_{1, h}^{\boldsymbol{\sigma}} \times M_{1, h}^{p}$, where $\tilde{M}_{1, h}^{\boldsymbol{\sigma}}:=\left\{\boldsymbol{\tau} \in M_{1, h}^{\boldsymbol{\sigma}}: \operatorname{div} \boldsymbol{\tau}=0\right.$ in $\Omega, \int_{\Omega} \operatorname{tr}(\boldsymbol{\tau})=0$, and $\left.\int_{\Omega} \boldsymbol{\delta}: \boldsymbol{\tau}=0 \forall \boldsymbol{\delta} \in M_{h}^{\boldsymbol{\gamma}}\right\}$

According to the definition of $M_{1, h}^{\boldsymbol{\sigma}}$, and using that $\operatorname{div}\left(\operatorname{curl}^{t} b_{T}\right)=0$, we deduce that the restriction of each element of $\tilde{M}_{1, h}^{\boldsymbol{\sigma}}$ to a triangle $T \in \mathcal{T}_{h}$ belongs to the local space $\left[\mathbf{P}_{0}(T)\right]^{2 \times 2} \oplus\left[\mathbf{P}_{0}(T) \mathbf{c u r l}^{t} b_{T}\right]^{2}$. In addition, similarly as for the nonlinear Stokes model, it is not difficult to realize that the conditions required to extend the proof of the continuous inf-sup condition for $\mathbf{B}_{1}$ to the discrete case, are given by

$$
\operatorname{dev}(\boldsymbol{\tau}) \in X_{1, h} \quad \text { and } \quad(-q \mathbf{I}+\boldsymbol{\tau}) \in X_{1, h} \quad \forall(\boldsymbol{\tau}, q) \in \tilde{M}_{1, h} .
$$

We observe again that there is no explicit restriction on $M_{1, h}^{p}$, and hence this subspace of $L^{2}(\Omega)$ is taken as the simplest possible one, that is

$$
M_{1, h}^{p}:=\left\{q \in L^{2}(\Omega):\left.\quad q\right|_{T} \in \mathbf{P}_{0}(T) \quad \forall T \in \mathcal{T}_{h}\right\}
$$


Next, taking into account the definitions of $\tilde{M}_{1, h}$ and $M_{1, h}^{p}$, we deduce that (35) is verified if $X_{1, h}$ is choosen so that its restriction on each triangle $T \in \mathcal{T}_{h}$ becomes the local space $\mathcal{E}_{0}(T):=\left[\mathbf{P}_{0}(T)\right]^{2 \times 2} \oplus\left[\mathbf{P}_{0}(T) \operatorname{curl}^{t} b_{T}\right]^{2} \oplus \operatorname{dev}\left(\left[\mathbf{P}_{0}(T) \operatorname{curl}^{t} b_{T}\right]^{2}\right)$, and therefore

$$
X_{1, h}:=\left\{\mathbf{s} \in\left[L^{2}(\Omega)\right]^{2 \times 2}:\left.\quad \mathbf{s}\right|_{T} \in \mathcal{E}_{0}(T) \quad \forall T \in \mathcal{T}_{h}\right\} .
$$

Finally, the unique solvability and stability of the Galerkin scheme associated with (30), the corresponding Cea estimate, and the rate of convergence, are established in a similar manner to Theorems 2.2 and 3.2. We omit details and refer to Theorems 4.1 and 4.2 in [16].

\section{$5 \quad$ Summary and further remarks}

The finite element subspaces providing the unique solvability and stability of the Galerkin schemes are summarized below in Table 5.1. We only list those spaces approximating the unknowns that are common to the three boundary value problems. We indicate the global regularity and local behaviour of the subspaces, and use the following notations to identify each problem: NSP (Nonlinear Stokes Problem), GSP (Generalized Stokes Problem), and NEP (Nonlinear Elasticity Problem).

Table 5.1: Finite element subspaces approximating the unknowns

\begin{tabular}{c|l|l}
\hline \multicolumn{1}{c|}{$\mathbf{\text { Global }}$} & Local \\
\hline $\mathrm{NSP}$ & {$\left[L^{2}(\Omega)\right]^{2 \times 2}$} & {$\left[\mathbf{P}_{0}(T)\right]^{2 \times 2}$} \\
$\mathrm{GSP}$ & {$\left[L^{2}(\Omega)\right]^{2 \times 2}$} & {$\left[\mathbf{P}_{0}(T)\right]^{2 \times 2} \oplus \operatorname{dev}\left(\left[\mathbf{P}_{0}(T) \mathbf{x}^{t}\right]^{2}\right)$} \\
$\mathrm{NEP}$ & {$\left[L^{2}(\Omega)\right]^{2 \times 2}$} & {$\left[\mathbf{P}_{0}(T)\right]^{2 \times 2} \oplus\left[\mathbf{P}_{0}(T) \mathbf{c u r l}^{t} b_{T}\right]^{2} \oplus \operatorname{dev}\left(\left[\mathbf{P}_{0}(T) \mathbf{c u r l}^{t} b_{T}\right]^{2}\right)$} \\
\hline $\boldsymbol{\sigma}$ & Global & Local \\
\hline $\mathrm{NSP}$ & $H(\mathbf{d i v} ; \Omega)$ & {$\left[\mathcal{R} \mathcal{T}_{0}(T)^{t}\right]^{2}$} \\
$\mathrm{GSP}$ & $H(\mathbf{d i v} ; \Omega)$ & {$\left[\mathcal{R} \mathcal{T}_{0}(T)^{t}\right]^{2}$} \\
$\mathrm{NEP}$ & $H(\mathbf{d i v} ; \Omega)$ & {$\left[\mathcal{R} \mathcal{T}_{0}(T)^{t}\right]^{2} \oplus\left[\mathbf{P}_{0}(T) \mathbf{c u r l}^{t} b_{T}\right]^{2}$} \\
\hline $\mathbf{u}$ & Global & Local \\
\hline All & {$\left[L^{2}(\Omega)\right]^{2}$} & {$\left[\mathbf{P}_{0}(T)\right]^{2}$} \\
\hline$p$ & Global & Local \\
\hline All & $L^{2}(\Omega)$ & $\mathbf{P}_{0}(T)$ \\
\hline
\end{tabular}


We observe that the above ordering corresponds to an increasing degree of enrichment (complexity) of the subspaces approximating the unknowns $\mathbf{t}$ and $\boldsymbol{\sigma}$. This is particularly notorious for $\mathbf{t}$, which, however, is atenuated by the fact that the subspaces are included in $\left[L^{2}(\Omega)\right]^{2 \times 2}$, and hence the local approximations can be handled independently. On the other hand, it is important to remark that, following the same approach employed here, the derivation of finite element subspaces of higher order is also possible.

Finally, we call the atention to the fact that reliable and quasi-efficient a-posteriori error estimates and the associated adaptive algorithms to compute the discrete solutions, are available for the dual-dual mixed variational formulations considered in this paper. To this respect, we mainly refer to [12] and [6] where the theoretical analysis and several numerical results illustrating the performance of the methods are reported.

\section{References}

[1] D.N. Arnold, F. Brezzi And J. Douglas JR., PEERS: A new mixed finite element method for plane elasticity. Japan Journal of Applied Mathematics, 1, (1984), 347367.

[2] D.N. Arnold, J. Douglas JR. And C.P. Gupta, A family of higher order mixed finite element methods for plane elasticity. Numerische Mathematik, 45, (1984), 1-22.

[3] Baranger, J., NAJib, K. And SAndri, D., Numerical analysis of a three-fields model for a quasi-Newtonian flow. Computer Methods in Applied Mechanics and Engineering, vol. 109, pp. 281-292, (1993).

[4] M.A. Barrientos, G.N. Gatica and E.P. Stephan, A mixed finite element method for nonlinear elasticity: two-fold saddle point approach and a-posteriori error estimate. Numerische Mathematik, 91, 2, (2002), 197-222.

[5] F. Brezzi, M. Fortin, Mixed and Hybrid Finite Element Methods. Springer Verlag, 1991.

[6] R. Bustinza, G.N. Gatica And M. GonzÁlez, A priori and a posteriori error analysis of a mixed finite element method for the generalized Stokes problem, submitted. Preprint 03-08, Departamento de Ingeniería Matemática, Universidad de Concepción (Chile), (2003).

[7] C. Carstensen and S. Funken, A-posteriori error control in low-order finite element discretisations of incompressible stationary flows. Mathematics of Computation, 70, 236, (2001), 1353-1381.

[8] G.N. GaticA, An application of Babuška-Brezzi's theory to a class of variational problems. Applicable Analysis, 75, 3-4, (2000), 297-303. 
[9] G.N. GaticA, Solvability and Galerkin approximations of a class of nonlinear operator equations. Zeitschrift für Analysis und ihre Anwendungen, 21, 3, (2002), 761-781.

[10] G.N. Gatica, L.F. Gatica, E.P. Stephan, A FEM-DtN formulation for a nonlinear exterior problem in incompressible elasticity. Mathematical Methods in the Applied Sciences, 26, 2, (2003), 151-170.

[11] G.N. Gatica, M. González and S. Meddahi, A low-order mixed finite element method for a class of quasi-Newtonian Stokes flows. Part I: a-priori error analysis, submitted.

[12] G.N. Gatica, M. González and S. Meddahi, A low-order mixed finite element method for a class of quasi-Newtonian Stokes flows. Part II: a-posteriori error analysis, submitted.

[13] Gatica, G.N. And Heuer, N., A dual-dual formulation for the coupling of mixedFEM and BEM in hyperelasticity. SIAM Journal on Numerical Analysis, vol. 38, 2, pp. 380-400, (2000).

[14] G.N. Gatica, N. Heuer and S. Meddahi, On the numerical analysis of nonlinear two-fold saddle point problems. IMA Journal of Numerical Analysis, 23, 2, (2003), 301-330.

[15] Gatica, G.N. And Meddahi, S., A dual-dual mixed formulation for nonlinear exterior transmission problems. Mathematics of Computation, vol. 70, 236, pp. 14611480, (2001).

[16] G.N. Gatica AND E.P. Stephan, A mixed-FEM formulation for nonlinear incompressible elasticity in the plane. Numerical Methods for Partial Differential Equations, 18, 1, (2002), 105-128.

[17] O. LADYZHENSKAYA, New equations for the description of the viscous incompressible fluids and solvability in the large for the boundary value problems of them. In Boundary Value Problems of Mathematical Physics V, AMS (Providence), 1970.

[18] Loula, A.F.D. ANd Guerreiro, J.N.C., Finite element analysis of nonlinear creeping flows. Computer Methods in Applied Mechanics and Engineering, vol. 79, pp. 87-109, (1990).

[19] J.E. Roberts And J.-M. Thomas, Mixed and Hybrid Methods. In Handbook of Numerical Analysis, vol. II, edited by P.G. Ciarlet y J.L. Lions, North-Holland (Amsterdam), 1991.

[20] SANDRI, D., Sur l'approximation numérique des écoulements quasi-Newtoniens dont la viscosité suit la loi puissance ou la loi de Carreau. Mathematical Modelling and Numerical Analysis, vol. 27, 2, pp. 131-155, (1993).

[21] B. SChEuRER, Existence et approximation de point selles pou certain problemes nonlinéaires. RAIRO Analyse Numérique, 11, 4, (1977), 369-400. 